of a similar nature. Thus we find the report of a bleeder admitted into the Sunderland Infirmary for synovitis of hip, who subsequently developed a tonsillar abscess, which on being opened caused almost fatal hæmorrhage. On inquiry it was found that the patient came of a hæmorrhagic stock. ${ }^{4}$ This probably was a case of hæmorrhagic effusion into the hip, as we learn that it quickly got well.

CASE 2. Supernumerary auricle on neck.-F. Thealthy boy, aged six months, was first seen on June 25th. On the left side of the neck, over the middle of the anterior border of the sterno-mastoid, was a nipple-like projection about half an inch in length; a rod of cartilage was prolonged into it from a thin plate which was freely movable in the subcutaneous tissue. This plate felt somewhat the shape of an auricle, being concave in front and terminating above and anteriorly in a sharp point. The projection had increased in size since birth. After a drawing had been made, the projection was removed by an elliptical incision. A process existed, dipping down deeply apparently beneath the sterno-mastoid, to which it was attached. The wound unfortunately suppurated, but healed quickly after a counter-opening had been made in the front of the neck. The condition found on dissecting the projection is shown in the accompanying drawing. The

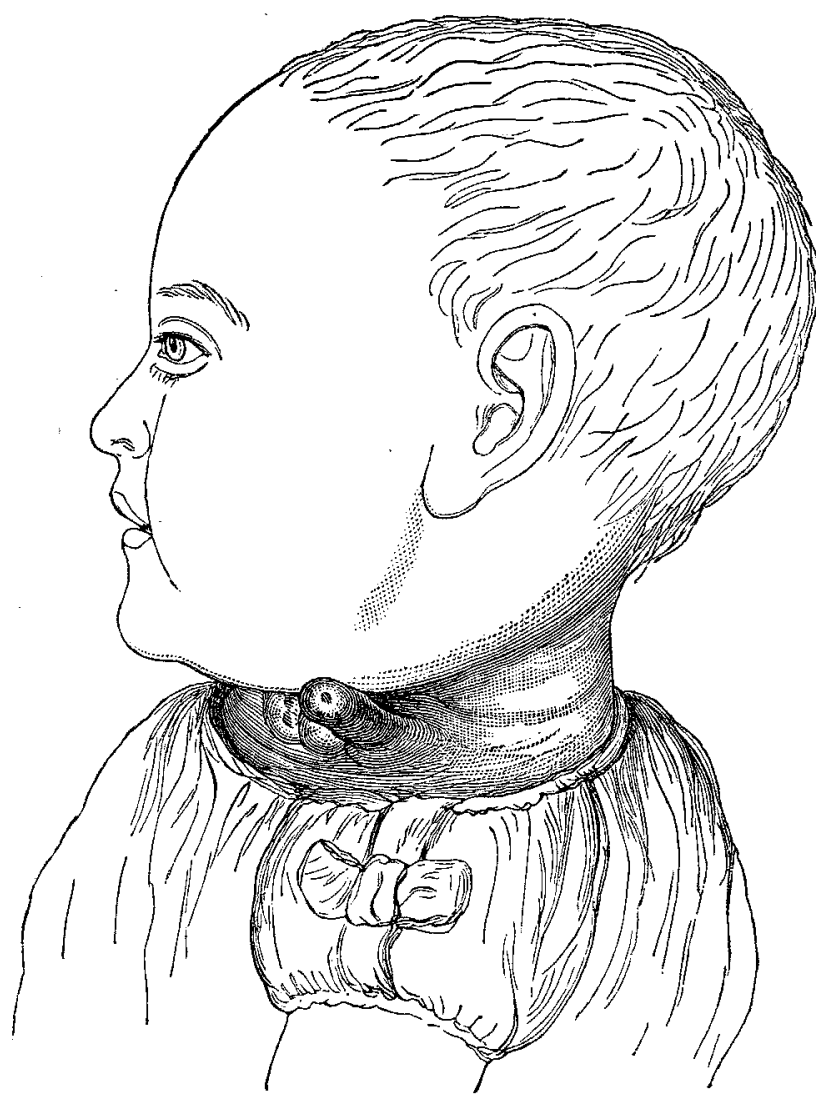

nipple-like nodule of skin contained a delicate rod of fibrocartilage, which is attached below to a eurved bar of bone, and this again terminates in a plate-like expansion, also of osseous material. The bar lay in the subcutaneous tissue, while the plate was deeply situated and was firmly attached to a muscle, probably the sterno-mastoid. A resemblance may easily be fancied between this structure and the pinna, the bar representing the anti-helix terminating below in the antitragus and the plate-like expansion the lobule. But unfortunately the deep situation and the osseous structure of these parts of the excised mass are at variance with the notion. The terminal rod of fibro-cartilage is the only part which could be considered the homologue of an auricle, while all that can be said of the deeper part is that it is a bar of bone probably formed in a branchial arch. To carry analogy further it may be compared to a hyoid or even a mandibular arch.

\section{ROYAL INFIRMARY, NEWCASTLE-ON-TYNE.} A CASE OF ATROPINE POISONING; REMARKS.

(Under the care of Dr. OLIVER.)

PoIsoning by atropine is not of frequent occurrence, and the following case is an important contribution to the literature on the subject. A dose of two grains of atropine has proved fatal in the adult. ${ }^{1}$ Dr. Oliver's patient appears to have taken nearly twice that amount, and the symptoms produced by the drug were severe. Drs. Ott and Colman ${ }^{2}$ found the increase of body temperature produced by atropine to be independent of the blood pressure, and believe its influence to be a stimulation of the thermogenetic centres in the spinal cord, considering that the rise of temperature is paralleled by that which occurs in tetanus. This case also adds one to the fairly large group in which the subcutaneous administration of morphia has apparently done good, a result contrary to the finding of the Edinburgh Committee, that "meconate of morphia does not act antidotally after a large dose of atropia; thus, whilst atropia is an antidote to morphia, morphia is not an antidote to atropia." For the following account we are indebted to Mr. T. W. Gibbard, house-physician.

M. A. D—, aged twenty-one years, was admitted into the Royal Infirmary, Newcastle-on-Tyne, on Aug. 4th, 1889, with symptoms of belladonna poisoning, having taken nearly one ounce of "eye-drops" (four grains of sulphate of atropine to the ounce) twelve hours previously, whilst drunk. Salt and water had been given by her friends, which had failed to produce vomiting. On admission, at 12.15 P.M., the patient's condition was as follows:-The face was flushed, the lips dry and blistered, tongue and mucous membrane of mouth dry. The eyes were somewhat prominent, the conjunctivæ injected, the pupils widely dilated and they did not react to light. There were twitchings of the fingers and toes, with marked tremor. She did not answer to her name, and refused to take milk. She was delirious, constantly moving in bed, picking the bedclothes, muttering, and pointing to imaginary objects. The movements were irregular and the skin dry. Temperature $101^{\circ} \mathrm{F}$., pulse 135 , respiration 32 (irregular). No vomiting. There was retention of urine, and the plantar and patellar reflexes were increased. She was ordered one ounce of castor-oil with eight minims of tincture of opium. At 3.30 P.M. the patient was still delirious and very excited; she did not appreciate questions and kept talking incoherently. There was no rash. Nearly forty ounces of urine, sp. gr. 1015, medium amber colour and free from albumen, were removed with difficulty by catheter, patient offering great resistance. She was given three minims of the hypodermic solution of morphia. At 6 P.M. she was not nearly so noisy. Free perspiration; temperature $100.4^{\circ}$. At 8 P.M. she was given another hypodermic injection of morphia, followed by four hours' sleep.

Aug. 5th.-Patient seems much better, talks quite sensibly, knows her name, and appreciates everything that is said to her; has taken milk. Her pupils are still dilated, tongue dry, and she complains of thirst. The skin is hot, temperature $103^{\circ}$, pulse 128 . Chest resonant on percussion and no abnormal sounds on auscultation over lungs. Heart sounds healthy. Has passed urine. Bowels have not acted. During the day she was very drowsy. Evening temperature $102^{\circ}$.

6th.-Morning temperature $100 \cdot 4^{\circ}$, evening $101^{\circ}$.

Still drowsy.

7 th.--Morning temperature $100^{\circ}$, evening $99 \cdot 8^{\circ}$; an enema was ordered, the bowels not having been opened since admission. The next day her temperature was normal and continued so, but she was still constipated, requiring a calomel and jalap powder on the 10th.

A week after admission the pupils were still dilated, though her sight was good. She was discharged well on the 16 th.

Remarks by Mr. GIBBARD. - I think the points of interest in this case are the following:-1. The high temperature for the first three days. This, in the absence of any inflammatory cause, confirms the opinion of most therapeutists as to the pyrogenic influence of atropine-vide Mitchell Bruce, who, however, regards the rise of temperature as correlated with the increased nutritive activity caused by quickened circulation and respiration. The heightened pulse and quickened respiration, noticeable before the rise of temperature, suggest that a large dose of poison was taken, a moderate dose being supposed to slow the pulse. 2. The constipation, which continued for a week after taking the poison, the patient requiring an enema on the 7 th and calomel on the loth, this being contrary to the general idea that belladonna has a tendency to cause in creased peristalsis of the bowel, and is probably explained|by

1 Taylor's Medical Jurisprudence, p. 433. 2 Wood's Therapeutics, p. 215. 
the peculiar effect of atropine upon the secretion of mucots $\mid$ a swelling was noticed in the right side of the abdomen. surfaces. 3. The good effect of morphia in this case, the skin becoming moist, and the delirium entirely disappearing after the second injection.

\section{WOLVERHAMPTON AND STAFFORDSHIRE GENERAL HOSPITAL.}

TWO CASES OF LITHOLAPAXY IN CHILDREN.

(Under the care of Mr. F. E. MANBY.)

WE presented our readers a fortnight ago ${ }^{1}$ with the records of four cases of removal of stone from the bladder in male children, two by means of litholapaxy and two by means of supra-pubic cystotomy. In the former the stones were of small size, weighing ten and thirty grains respectively, and in the latter 215 and 562 grains. The following cases are equally good examples of the successful crushing and removal of small stones, and resemble them in many particulars. It is advisable that the surgeon be provided with a second lithotrite, as one may get out of order or break. For the following notes we are indebted to Mr. J. Harley Gough, late house-surgeon.

CASE 1.- Ernest P - aged five, was admitted into the hospital on Feb. 4th, 1889, suffering from symptoms of stone in the bladder. The boy was sounded and a small calculus detected. On March 8th litholapaxy was performed under chloroform, a No. 5 lithotrite being used to crush the stone, and the débris evacuated with a Thompson's bladder aspirator and No. 6 cannula. The patient soon recovered from the effects of the anæesthetic, and towards evening was playing in bed apparently quite well. He passed urine freely, and only coniplained at first of a slight scalding sensation in the urethra. There was some little sandy deposit in the urine for the first two days. On March 17th he was sounded under chloroform very carefully, but nothing abnormal was detected in the bladder. He was discharged cured on March 24th. The stone was composed of uric acid, and the dried fragnents weighed nine grains.

CASE 2.-Frank $R$ - aged nine, was admitted on July 29th, 1889, with very severe symptoms of vesical calculus, and when sounded a moderate-sized calculus was detected. On July 25th the stone was crushed under chloroform with a No. 5 lithotrite, and the fraginents evacuated as in the preceding case. The operation lasted about forty minutes. The patient soon rallied from the effects of the operation, and expressed himself much relieved, all his urinary troubles having disappeared. On July 28th he was sounded under chloroform again, and with negative results. The boy was discharged cured the next day. The calculus was a uric acid one, with an external layer of phosphates; the dried fragments weighed thirty-six grains. These boys were seen recently, and the parents of each say they have been quite well, and have had no bad symptoms since they left the hospital.

\section{atteoiral Sorieties.}

\section{ROYAL MEDICAL \& CHIRURGICAL SOCIETY}

\section{Ruptured Tubal Pregnancy.}

AN ordinary meeting of this Society was held on Nov. 12th, the chair being occupied by the President, Sir Edward Sieveking.

Mr. Bland SutTon communicated a paper on a case of Ruptured Tubal Pregnancy, and added some remarks on the cause of early rupture. The patient was a woman aged thirty-seven, who, though married for seventeen years, had never before been pregnant. There was no history of gonorrhcea. The catamenia had been regular till three months before admission, when she missed two periods. Five weeks before admission the patient was attacked with collapse, from which she slowly recovered; a few days later

1 The LAycet, Mirror of Hospital Practice, vol. ii., p. 903.
On admission into the Middlesex Hospital a swelling was found in the right iliac fossa, which extended upwards to the costal arch and inwards to the mid-line. A sound introduced into the uterus passed to the extent of three inches. In Douglas's fossa on the right side a swelling was felt behind the uterus ; there was tenderness over the abdominal aspect of this swelling. The temperature for the twelve succeeding days varied between $99^{\circ}$ and $101^{\circ} \mathrm{F}$. Laparotomy was performed, and putrid blood clot was found in the pelvis extending up to the liver. Smart bleeding from the right broad ligament occurred, which was checked by transfixion of the ligament close to the uterus. The tube and ovary were removed, the abdomen washed out with warm water, and a glass drainage tube inserted for three days. The patient did well. The ovary contained a true corpus luteum of pregnancy, and a rounded FIG. 1.

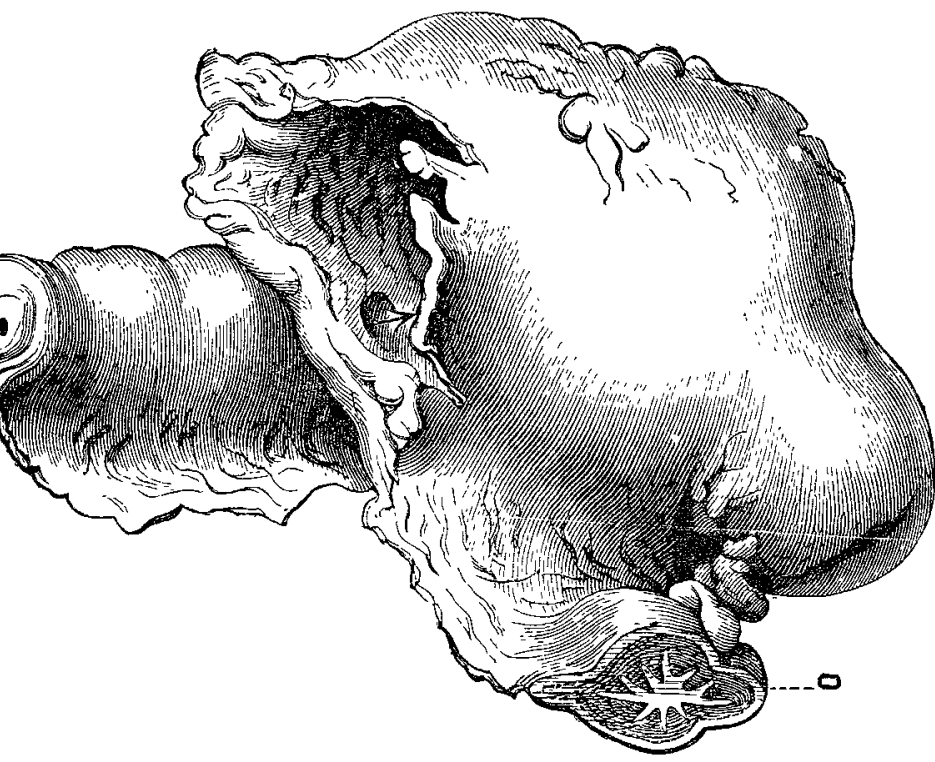

The distended Fallopian tube, showing the situation of the rupture. $o$, Ovary with corpus luteum.

mass removed contained an apoplectic ovum in which was a fcetus of the seventh or eighth week. The varieties of FIG. 2.

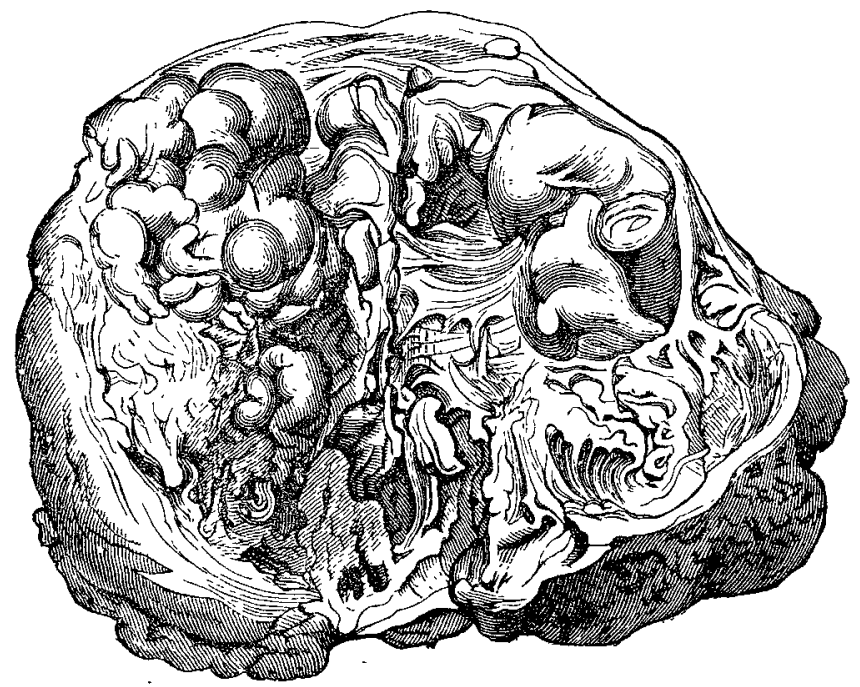

Apoplectic ovum from the Fallopian tube, represented in Fig. I.

apoplectic ovum were then discussed, and it was suggested that if an ovum of the size of a walnut became suddenly enlarged by hæemorrhage to that of an orange the result would be expulsion if it lay in the nterus, but rupture with hæmorrhage if it lay in the tube. The author urged finally that early rupture was to be attributed to the formation of an apoplectic ovum suddenly distending the tube, and held that no intra-peritoneal hæmatocele should be attributed to ruptured tubal pregnancy unless membranes, a fotus, or both were forthcoming, however suggestive the clinical evidence might be.

FIG. 3.

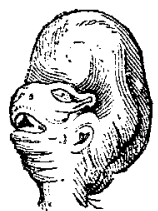

Distorted head of the foetus. About seventh or eighth week of gestation. (Figs. 1, 2, 3.) It would also be valuable to obtain an 